\title{
Avaliação da síndrome de burnout em servidores da guarda municipal de Fortaleza
}

Evaluation of the burnout syndrome among servers in Fortaleza municipal guard

\author{
T. H. R. Q. MOURÃO ${ }^{1 *}$; C. F. MELO \\ ${ }^{I}$ Curso de Psicologia, Universidade Estadual do Ceará (UECE), 60.714-903, Fortaleza - Ceará, Brasil \\ ${ }^{2}$ Pós-graduação em Psicologia, Universidade de Fortaleza (UNIFOR), 60.811-905, Fortaleza - Ceará, Brasil \\ *thais.helena1401@gmail.com
}

(Recebido em 21 de novembro de 2016; aceito em 27 de março de 2017)

\begin{abstract}
O profissional de defesa civil, que possui um trabalho que sempre foi considerado nobre, sofre com os custos emocionais eminentes de sua profissão, o que pode resultar em agravos como a síndrome de burnout, um esgotamento profissional. Desse modo, a presente pesquisa objetiva avaliar se os profissionais da Guarda Municipal de Fortaleza (GMF) apresentam a síndrome de burnout. Por meio de um estudo correlacional contou-se com uma amostragem não probabilística por conveniência composta por 147 servidores da GMF que responderam a Escala Maslach Burnout Inventory - Human Service Survey (MBI - HSS) e um questionário sociodemográfico. Os dados foram analisados por meio de estatística descritiva, com apoio do software Statistical Packageof Social Sciences for Windows (SPSS). Como resultados, verificou-se que os guardas municipais apresentam índices moderados com sinal de alerta de exaustão emocional $(M=22,23 ; \mathrm{DP}=14,55)$, despersonalização $(M=8,15 ; \mathrm{DP}=7,69)$ e realização profissional $(\mathrm{M}$ $=32,63 ; \mathrm{DP}=10,33)$. Contempla-se ainda que 35 participantes $(23,80 \%)$ apresentam a combinação de altos níveis de exaustão emocional e despersonalização. Desses, 26 participantes $(17,70 \%)$ apresentam, além dos altos níveis de exaustão emocional e despersonalização, baixo nível de realização profissional; configurando-se o quadro de burnout. Nessa perspectiva, conclui-se que os profissionais da guarda municipal apresentam burnout ou risco de adoecimento, fazendo-se necessárias intervenções de prevenção e recuperação.

Palavras-Chave: esgotamento profissional; burnout; guarda municipal.
\end{abstract}

The civil defense professional, who has a job that has always been considered noble, suffers with the eminent emotional costs of his profession, which can result in such problems as burnout syndrome, a professional exhaustion. Thus, the present study aims to evaluate if the professionals of the Municipal Guard of Fortaleza (GMF) present the burnout syndrome. A non-probabilistic convenience sample consisted of 147 GMF servers that responded to the Maslach Burnout Inventory-Human Service Survey (MBI-HSS) and a sociodemographic questionnaire. The data were analyzed through descriptive statistics, with the support of Statistical Package of Social Sciences for Windows (SPSS). As results, it was verified that the municipal guards present moderate indexes with alert sign of emotional exhaustion $(\mathrm{M}=22.23, \mathrm{SD}$ $=14.55)$, depersonalization $(\mathrm{M}=8.15, \mathrm{SD}=7.69)$ and achievement $(\mathrm{M}=32.63, \mathrm{SD}=10.33)$. It is also contemplated that 35 participants $(23.80 \%)$ present a combination of high levels of emotional exhaustion and depersonalization. Of these, 26 participants (17.70\%) present, in addition to the high levels of emotional exhaustion and depersonalization, low level of professional achievement; Setting the burnout frame. From this perspective, it is concluded that the municipal guard professionals the burner or risk of becoming ill, making prevention and recovery interventions necessary.

Keywords: professional exhaustion; burnout; municipal guard.

\section{INTRODUÇÃO}

O trabalho é considerado vital para a construção do indivíduo, pois ele ocupa o papel de categoria fundamental para entender a totalidade dos complexos que constituem o ser social, além de proporcionar a transformação do homem e de sua sociedade [1]. Por outro lado, ele também 
pode gerar adoecimento, inclusive sobre a saúde mental, sendo um facilitador e promotor de fobias, ansiedades, depressão, eventos pós-traumáticos e somatizações [2].

Isso se deve, principalmente, ao modelo atual de organização do trabalho, que privilegia o aumento da produtividade, em detrimento da saúde mental do trabalhador. Realidade agravada pelo contexto atual de instabilidade dos vínculos empregatícios, precarização do trabalho e sentimento de insegurança frente ao desemprego estrutural [3], bem como os problemas de gestão, inadaptação e estresse envolvido em algumas atividades [4,5]. Para representação desse quadro, os levantamentos realizados por Da Silva - Junior e Fischer [6] relatam que o adoecimento mental foi a terceira principal causa de concessão do auxílio-doença pelo Instituto Nacional do Seguro Social (INSS) em 2008, atingindo 10,70\% dos casos. Acerca da incidência de cada patologia, detectou-se que as maiores incidências relacionadas ao trabalho entre 2008 e 2010 foram os transtornos do humor, como a depressão (quadro caracterizado pela redução de humor e outros sintomas). No entanto, em 2011, assumem a maior incidência os transtornos relacionados ao "stress" e os somatoformes (quadro caracterizado por sintoma físico não completamente explicado por condição médica geral).

Dentre os problemas que podem ocorrer, principalmente entre os profissionais que atuam com proximidade ao público, é a síndrome de burnout. Um quadro difícil de diagnosticar e, por vezes confundido com depressão. É caracterizado pela sensação de estafa ou fadiga extrema, que ocorre em consequência a uma exposição exacerbada a estressores emocionais e relacionais que são constantemente verificados na rotina de trabalho. Assim, no trabalho que antes o profissional apresentava-se vinculado afetivamente à função desenvolvida, pode, ao longo do tempo, ter essa relação desgastada, até que se chega ao extremo do esgotamento. Nesse contexto, o sentido do trabalho se esvai, transformando-se em uma sobrecarga, o que reflete em apatia e desinteresse pelo trabalho $[7,8]$.

Igualmente, o profissional de segurança pública, que possui um trabalho historicamente considerado nobre, por poder ajudar o outro e fazer a proteção pública, sofre com os custos emocionais eminentes de sua profissão. O exercício de sua função, que envolve ações com riscos proeminentes, pelo fato de lidarem cotidianamente com a violência e a criminalidade; traz aspectos potencializadores de adoecimento. Ademais, enfrentam adversidades vinculadas às condições de trabalho, como carga horária exaustiva e escassez de recursos e instrumentos laborais, que agravam esse panorama $[9,10]$. Esses fatores, quando apresentados de modo conjunto e continuado, fazem com que esses profissionais acumulem cargas emocionais exaustivas, fazendo com que o trabalho, que antes era percebido como nobre e prazeroso, agora gere esgotamento. Realidade que pode vir a interferir na sua atuação junto aos cidadãos, como também abranger outros âmbitos de sua vida [11].

$\mathrm{Na}$ abordagem sobre essa problemática, Guimarães et. al. [12], em estudo sobre a incidência de síndrome de burnout (SB) em policiais militares (PM) e civis (PC) na cidade de Campo Grande - MS, verificaram que policiais civis e militares apresentam elevados índices de burnout. Índices levemente maiores entre os PC, principalmente nos critérios que consideravam a exaustão emocional e a diminuição do envolvimento pessoal.

Igualmente um estudo com os agentes penitenciários, profissionais que convivem com modalidades diversas de violência e assujeitamento, afetando sua saúde mental, verificou-se a existência de alguns casos com quadros de ansiedade paranóide e anomia, entre outros fenômenos psicossomáticos [13]. De modo semelhante, estudos realizados com guardas municipais mostram que algumas especificidades dessa categoria podem ser geradoras de sofrimentos. Como principal causa, aponta-se a indefinição dos serviços a serem praticados pela categoria que, apesar de terem a função de se responsabilizar pela preservação de bens e patrimônios públicos, em alguns casos são incumbidos de outras funções [14]. Ademais, os guardas municipais também destacam a falta de índices de produção que avaliem a efetividade de sua prática, já que o trabalho realizado por essa categoria se configura como imaterial, que neste caso, envolve os afetos e interações humanas, interfere na subjetivação do trabalhador e dificulta o dimensionamento de sua atuação $[14,15]$.

Diante desse panorama, principalmente no tocante à promoção de saúde do trabalhador e da avaliação de condições laborais, as produções acerca da síndrome de burnout vêm sendo enfatizadas. Dessa forma, compreende-se como essencial abranger nesses estudos os profissionais 
que atuam na segurança pública, especialmente na cidade de Fortaleza, considerada a mais violenta do país, onde estes profissionais, apesar de muitas vezes não serem percebidos pela população e não haver campanhas de apresentação e divulgação de seu trabalho, possuem uma função arriscada e estressante. Diante do exposto, o presente estudo objetivou avaliar se os profissionais da Guarda Municipal de Fortaleza (GMF) apresentam burnout, através de um levantamento do perfil dos participantes, da identificação dos critérios de burnout; e de um estudo de correlação entre as variáveis biodemográficas e da síndrome de burnout na amostra estudada.

\section{MATERIAL E MÉTODOS}

\section{Delineamento}

A atual pesquisa define-se como um estudo correlacional no qual as variáveis primárias vinculam-se às condições de trabalho na guarda municipal de Fortaleza e as variáveis consequentes relacionam-se aos índices de ocorrência da síndrome de burnout, verificados entre os profissionais.

\section{Locus da Pesquisa}

A Guarda Municipal de Fortaleza (GMF) é a instituição responsável pela segurança pública do município de Fortaleza, Ceará. Apresenta como atribuições de trabalho a proteção do patrimônio público ecológico, arquitetônico e cultural do município; além de desenvolver ações de prevenção à violência, utilizando como estratégias de atuação as medidas educativas e promotoras de diálogo. De forma paralela, atua conjuntamente com a Defesa Civil, instituição que desenvolve ações, programas e projetos vinculados à prevenção, apoio e recuperação da comunidade em situação de vulnerabilidade social, decorrentes de pouca infraestrutura, alterações climáticas, remoções, entre outros fatores. Atua, desse modo, em prol de reduzir os impactos ocasionados por essas variáveis e restabelecer a normalidade social [16].

\section{Amostra da Pesquisa}

O número total de servidores da GMF é de cerca de 1.000 guardas municipais. Para um valor de nível de confiança de $95 \%$ e de erro amostral de 5\%, contou-se com uma amostra não probabilística por conveniência de 147 servidores.

\section{Instrumento}

Utilizou-se a Escala Maslach Burnout Inventory - Human Service Survey (MBI - HSS) [17], traduzida e adaptada por Lautert [18]. A escala é composta por 22 afirmações, cuja as respostas são atribuídas em uma escala de pontuação no modelo Likert, variando de 0 a 6 . Os itens são distribuídos em três dimensões relacionadas à síndrome de burnout: a exaustão emocional, abrangendo 9 afirmações $(1,2,3,6,8,13,14,16,20)$; a despersonalização, vinculada a 5 afirmações $(5,10,11,15,22)$; e a realização profissional, com 8 afirmações $(4,7,9,12,17,18$, 19, 21). Além disso, utilizou-se um questionário sociodemográfico, com intuito de relacionar os resultados encontrados sobre sexo, idade, tempo de serviço e cargo ocupado. Ademais, foi utilizado diário de campo, para a apreensão das observações e dos relatos coletados.

\section{Procedimentos Éticos e de Coleta de Dados}

Após aprovação da pesquisa pela diretoria da GMF e por comitê de ética da Universidade Estadual do Ceará, sob o protocolo $\mathrm{n}^{\circ} 0298$, foram realizadas visitas sistemáticas à sede da GMF para conhecimento do campo de pesquisa e contato com seus profissionais. Após aceite dos participantes, os instrumentos foram aplicados de forma individual e no modelo autoaplicável, na 
sede da GMF e em pontos externos de trabalho da GMF. Os participantes da pesquisa foram informados, de modo prévio, dos objetivos e procedimentos do estudo, lhes garantindo o anonimato em sua colaboração e a confidencialidade de suas respostas. Ademais, solicitou-se aos participantes que assinassem um Termo de Consentimento Livre e Esclarecido (TCLE), de acordo com a "Resolução N. 266/12 sobre Pesquisa Envolvendo Seres Humanos".

\section{Procedimentos de Análise de Dados}

Os dados foram analisados por meio de estatística descritiva, com auxílio do pacote estatístico Statistical Packageof Social Sciences for Windows (SPSS), versão 21, em duas etapas. Inicialmente, os níveis de burnout dos profissionais da GMF foram analisados baseados na normatização estabelecida pelos autores do MBI - HSS. Para tanto, é considerado o escore total para cada dimensão, por meio do somatório de pontuações de seus itens: em Exaustão Emocional, com 9 itens, as pontuações variam entre 0 e 54 pontos; em Despersonalização, com 5 itens, as pontuações variam entre 0 e 30 pontos; e em Reduzida Realização Pessoal, com 8 itens, as pontuações variam entre 0 e 48 pontos. Deve também ser considerada a distribuição dessas pontuações nos estratos de classificação dos níveis de burnout (ver Tabela 1). Em seguida, foram realizadas comparações dos índices de burnout por variáveis sociodemográficas.

Tabela 1. Padrão de pontuação para classificação dos níveis de burnout por dimensões do MBIHSS

\begin{tabular}{llll}
\hline Fator & \multicolumn{3}{c}{ Níveis de burnout } \\
\cline { 2 - 4 } & Baixo & Moderado & Alto \\
\hline Exaustão Emocional & $0-17$ pontos & $18-31$ pontos & $32-54$ pontos \\
Despersonalização & $0-03$ pontos & $4-10$ pontos & $11-30$ pontos \\
Realização Profissional & $0-30$ pontos & $31-37$ pontos & $38-48$ pontos \\
\hline
\end{tabular}

\section{RESULTADOS E DISCUSSÃO}

\section{Características biodemográficas da amostra de guardas municipais}

Entre os 147 guardas da amostra do estudo, existem 56 mulheres $(38,10 \%)$ e 91 homens $(61,90 \%)$. Eles possuem em média 34,41 anos $(\mathrm{DP}=8,45)$, sendo o mais novo com 26 anos e o mais velho com 67 anos. Atuam na guarda municipal em média há 10,11 anos (DP =6,80), com variações entre 5,58 e 48 anos. Na distribuição das funções, 85 são guardas operacionais $(57,80 \%)$, que atuam de forma ostensiva no município; e 62 são guardas administrativos $(42,20 \%)$, que se responsabilizam pelos recursos administrativos e burocráticos.

\section{Avaliação dos índices de burnout nos guardas municipais}

Os resultados mostram que, de modo geral, os guardas municipais apresentam moderada exaustão emocional $(\mathrm{M}=22,23 ; \mathrm{DP}=14,55)$, moderada despersonalização $(\mathrm{M}=8,15 ; \mathrm{DP}=7,69)$ e moderada realização profissional $(\mathrm{M}=32,63$; $\mathrm{DP}=10,33)$, sugerindo que nas três dimensões há problemas com a saúde mental dos profissionais, indicando sinal de alerta para risco de burnout. Dados que entram em concordância com o panorama trazido por Cabral e Zeni [19], que em pesquisa sobre qualidade de vida com guardas municipais da cidade de Chapecó - SC perceberam a influência de aspectos relacionados às atividades laborais na ocorrência de sofrimento mental desses trabalhadores.

De forma mais específica, os índices de exaustão emocional variaram entre 0 e 54 escores. Entre os participantes, $66(44,89 \%)$ apresentaram baixa exaustão emocional, $37(25,17 \%)$ apresentaram moderada exaustão emocional e 44 (29,93\%) apresentaram alta exaustão 
emocional. Desse modo, verifica-se que cerca de 55\% dos servidores encontra-se com níveis de exaustão entre moderado e alto. Esse critério da síndrome de burnout sinaliza a existência de um esgotamento emocional e físico, como uma carência de energia, podendo estar vinculado ao contexto laboral desses profissionais. Estudos relatam que esses fatores aversivos verificados no trabalho são originadores de diversas patologias, entre eles, os transtornos depressivos e de ansiedade [20]. Realidade apresentada também em 12\% dos militares da Guarda Nacional Republicana Portuguesa [21]; em seguranças portugueses $-25,50 \%$ nas forças de segurança prisional e $11.80 \%$ nas forças de segurança pública [22] e em policiais civis do Rio Grande do Sul [23]

Os índices de despersonalização variaram entre 0 e 29 escores. Entre os participantes, 52 $(35,37 \%)$ apresentaram baixa despersonalização, 49 (33,33\%) apresentaram moderada despersonalização e 46 (31,29\%) apresentaram alta despersonalização. Esses dados demonstram que mais de $60 \%$ dos guardas municipais de Fortaleza apresentam limitações e dificuldades relacionadas ao convívio com seus pares e a população de forma geral, aspectos que caracterizam a despersonalização. Masumoto e Faiman [5] verificaram que este processo ocorre à medida que estes sujeitos tendem a criar formas de enfrentamento e proteção aos aspectos laborais que lhes são problemáticos, no entanto, esse modelo de percepção do contexto pode ser transferido às outras relações e afetar os vínculos desses trabalhadores. Dados confirmados na literatura em 10\% dos militares da Guarda Nacional Republicana Portuguesa [21]; em seguranças portugueses $20,90 \%$ nas forças de segurança prisional e $6.50 \%$ nas forças de segurança pública [22] e em policiais civis do Rio Grande do Sul [23]

Os índices de realização profissional variaram entre 8 e 48 escores. Entre os participantes, 52 $(35,37 \%)$ percebiam-se como pouco realizado profissionalmente, $49(33,33 \%)$ sentiam moderada realização e $46(31,29 \%)$ apresentaram alta realização. Neste aspecto, quase $70 \%$ dos servidores apresentaram insatisfações em relação à efetividade de sua prática profissional. Devido à especificidade da atuação da guarda, que age na prevenção da violência e promoção de segurança, os produtos de seu trabalho são dificilmente quantificados, gerando assim um sentimento de ineficácia ou improdutividade. Atualmente, muitos profissionais que lidam com essa modalidade de trabalho, denominado imaterial, apresentam como sintomatologia a sensação de baixa ou moderada realização profissional, em grande parte, por causa da pouca valorização profissional e por lidar diariamente com os afetos e cobranças sociais [15]. Realidade também vivenciada por 8\% dos militares da Guarda Nacional Republicana Portuguesa [21]; em seguranças portugueses $-3,40 \%$ nas forças de segurança prisional e $7.60 \%$ nas forças de segurança pública [22] e em policiais civis do Rio Grande do Sul [23]

Tabela 2. Distribuição dos participantes por níveis de burnout em cada dimensão do MBI - HSS

\begin{tabular}{llll}
\hline Fator & \multicolumn{3}{c}{ Níveis de burnout } \\
\cline { 2 - 4 } & Baixo & Moderada & Baixo \\
\hline Exaustão & $f=66(44,90 \%)$ & $f=37(25,17 \%)$ & $f=44(29,93 \%)$ \\
Despersonalização & $f=52(35,38 \%)$ & $f=49(33,33 \%)$ & $f=46(31,29 \%)$ \\
Realização Profissional & $f=52(35,38 \%)$ & $f=49(33,33 \%)$ & $f=46(31,29 \%)$ \\
\hline
\end{tabular}

Contempla-se ainda que 35 participantes $(23,80 \%)$ apresentam de forma concomitante altos índices de despersonalização e exaustão emocional. Desses, 26 participantes $(17,70 \%)$ apresentam, além dos altos níveis nos critérios citados, um baixo nível de realização profissional; configurando-se um quadro de burnout. Desse modo, verifica-se que a incidência de burnout em 26 servidores da GMF é considerável, pois, embora aparentemente seja uma porcentagem pequena, durante analise dos três aspectos componentes do quadro de burnout encontram-se taxas mais elevadas, quando comparadas a outros contextos laborais de instituições de segurança pública. Como conseguinte, faz-se necessária uma avaliação das questões laborais problemáticas, já que estes servidores, em grande parte, atuam junto à população em ações promotoras de segurança e cuidado. 


\section{Comparações dos índices de burnout por características sociodemográficas}

É possível verificar ainda que os índices de burnout apresentaram diferenças nas comparações por dados sociodemográficos. A seguir são apresentadas apenas as avaliações que se destacaram pela existência de diferenças estatisticamente significativas.

Verificou-se que houve diferenças estatisticamente significativas nas comparações dos índices de burnout por tipo de cargo ocupado. O fator exaustão emocional apresentou diferenças significativas $\left[\chi^{2}(2, N=145)=204,83, p<0,05\right]$, onde os guardas operacionais apresentaram maiores pontuações $(\mathrm{M}=24,69$; $\mathrm{DP}=16,06)$ que os administrativos $(\mathrm{M}=18,85$; $\mathrm{DP}=11,48)$. $\mathrm{Na}$ despersonalização também foram observadas diferenças nas comparações por cargo $\left[\chi^{2}(2, N\right.$ $=145)=97,84, p<0,05]$, onde os guardas operacionais apresentaram maiores pontuações $(\mathrm{M}=$ 10,03; $\mathrm{DP}=8,60)$ que os administrativos $(\mathrm{M}=5,56$; $\mathrm{DP}=5,27)$. Ademais, estas discrepâncias também foram percebidas em análise do fator realização profissional nas comparações por cargo $\left[\chi^{2}(2, N=145)=54,57, p<0,05\right]$, onde os guardas operacionais apresentaram menores pontuações $(M=30,00 ; \mathrm{DP}=11,50)$ que os administrativos $(\mathrm{M}=36,24 ; \mathrm{DP}=7,09)$. Desse modo, constata-se que há diferenças na incidência dos sintomas de burnout de acordo com a modalidade de atuação. Variação esta possivelmente relacionada às diferentes especificidades dessas atribuições, já que os servidores operacionais atuam diretamente com a população e tendem a lidar com as problemáticas sociais continuamente, somam-se a esse contexto as baixas condições de trabalho, como falta de equipamentos e de locais apropriados para a realização das atividades laborais [14].

Verificou-se ainda a ocorrência de diferenças estatisticamente significativas nas comparações dos índices de burnout por idade. $\mathrm{O}$ fator exaustão emocional apresentou diferenças significativas $\left[\chi^{2}(3, N=145)=204,05, p<0,05\right]$, onde os guardas mais novos (26-35 anos) destacam-se por apresentaram maiores pontuações $(\mathrm{M}=25,07 ; \mathrm{DP}=14,07)$ que os guardas de idade mediana (3649 anos $)(M=18,56 ; \mathrm{DP}=14,14)$ e mais velhos $(50-67$ anos $)(\mathrm{M}=18,50 ; \mathrm{DP}=16,08)$. Nas comparações por tempo de serviço, também se percebeu diferenças estatisticamente significativas no fator realização profissional $\left[\chi^{2}(3, N=145)=104,78, p<0,05\right]$, onde os guardas com menor tempo de serviço (5-10 anos) destacam-se por apresentaram menores pontuações $(\mathrm{M}=30,93$; DP $=10,74)$ que os guardas que atuam entre $11-20$ anos $(\mathrm{M}=35,94 ; \mathrm{DP}=8,62)$ e $21-48$ anos $(\mathrm{M}=$ $35,92 ; \mathrm{DP}=10,09)$. Essas diferentes relações com o trabalho também puderam ser percebidas nas falas dos servidores enquanto os questionários eram aplicados, pois se averiguou que, entre os guardas de idades mais avançadas e os que tinham maiores tempos de serviço, havia maior identificação com o trabalho e a construção de um sentimento de identidade com as atividades desenvolvidas. Desse modo, eles tendiam a encarar a sua atuação de forma mais significativa e com mais sentido em seu contexto, assim a identidade surge como um fator de saúde mental [14].

Em resumo, os resultados trazidos demonstram que são verificados neste público índices significativos em relação aos fatores que compõem o quadro característico de burnout, com incidência em 17,70\% dos guardas municipais. Nesse panorama, Masumoto e Faiman [5] reforçam que, entre os fatores propiciadores no adoecimento, se destacam a pressão e sobrecarga de atividades, falta de apoio e autonomia no trabalho, a falta de convívio com familiares e amigos e o assedio moral. Já entre os fatores de proteção estariam a valorização profissional, o respeito, o apoio de colegas e chefes e a promoção de autonomia. Considerando essas potencialidades e limitações, faz-se necessário que os gestores municipais reconheçam que a saúde mental dos profissionais da segurança pública incide sobre a qualidade do seu trabalho e em sua relação com a comunidade. Torna-se, portanto, importante a realização de contínuas intervenções de cuidado à saúde para estes profissionais, a fim de que uma atuação eficaz e qualificada surja como retorno à população. Pois embora a instituição tenha um serviço direcionado à prevenção e promoção de saúde, o Núcleo Biopsicossocial (NUSOCI), este deveria contar com equipe profissional mais ampla e atuar de forma conjunta com a direção da GMF, já que todas as ações e intervenções planejadas por ambos devem estar em consonância, a fim de possibilitar maio participação dos servidores e menores índices de adoecimento. 


\section{CONCLUSÃO}

As relações de trabalho vêm continuamente sendo alteradas. De modo paralelo, as temáticas de flexibilização dos vínculos empregatícios e precarização do trabalho são incessantemente debatidas e pesquisadas. Esse contexto de insegurança e instabilidade afeta diretamente os trabalhadores, o que se verifica por meio dos crescentes estudos acerca da síndrome de burnout e outros transtornos que possuem nexo causal com o trabalho. Aliado a esse panorama, se verifica um crescente número nos índices de violência e criminalidade, que fortalecem os medos e angústias sociais.

Esses fatores se interligam com a atuação dos guardas municipais fortalezenses, pois além das condições adversas inerentes à rotina de trabalho, estes ainda lidam com os afetos e sentimentos da população, que anseia por mais intervenções que intensifiquem a segurança pública. Desse modo, este estudo pretendeu analisar quais as consequências dessas variáveis na saúde mental desses profissionais, mais especificamente, se poderia ser verificada alguma incidência de síndrome de burnout na GMF.

Entre os dados encontrados percebeu-se uma incidência pequena de casos de Burnout entre os servidores, embora no tocante aos aspectos componentes da síndrome (despersonalização, exaustão emocional e baixa realização profissional) tenham sido verificados índices maiores. Usando como parâmetro de avaliação o comparativo com outras categorias profissionais vinculadas à segurança pública, conclui-se que é um quantitativo considerável, por tratar-se de um âmbito de atuação profissional que lida diretamente com a proteção e cuidado da população.

Em termos gerais, reconhece-se a limitação do presente estudo por utilizar uma amostra não probabilística e local e com coleta de dados transversal. Assume que é deixado aqui uma pesquisa exploratória que possa subsidiar uma investigação mais ampla de avaliação do cenário de saúde mental dos profissionais da guarda municipal a nível municipal e nacional, compreendendo as particularidades do trabalho do guarda que podem contribuir com o surgimento do burnout, de forma a fundamentar medidas de prevenção mais eficazes.

\section{REFERÊNCIAS BIBLIOGRÁFICAS}

1. Santos Neto AB. Trabalho e tempo de trabalho na perspectiva marxiana. 1 ed. São Paulo: Instituto LUCKACS; 2013. 168 p.

2. Dejours C. A loucura do trabalho: Estudo de psicopatologia do trabalho. 5 ed. Paraguay AI, Ferreira LL, tradutoras. São Paulo: Cartaz - Oboré; 1992. 173 p.

3. Bauman Z. A sociedade individualizada: vidas contadas e histórias vividas. Gradei J, tradutor. Rio de Janeiro: Jorge Zahar; 2008. 164 p.

4. Druck G. Trabalho, Precarização e Resistências: novos e velhos desafios? . Caderno CRH. 2011; 24(1): 37-57, doi: 10.1590/S0103-49792011000400004.

5. Masumoto LK, Faiman CJS. Saúde mental e trabalho: um levantamento da literatura nacional nas bases de dados em Psicologia da Biblioteca Virtual de Saúde (BVS). Saúde, Ética \& Justiça. 2014; 19(1): 1 11, doi: 10.11606/issn.2317-2770.v19i1p1-11.

6. Da Silva Junior JS, Fischer FM. Adoecimento mental incapacitante: benefícios previdenciários no Brasil entre 2008-2011. Rev Saúde Pública. 2014; 48(1): 186 - 190, doi: 10.1590/S00348910.2014048004802.

7. Gomes SR, Gomes AR. Diagnósticos de enfermagem relacionados à síndrome de burnout. REINPEC. 2015; 2 (1): 60 - 71, doi: 10.20951/2446-6778/v1n2a5.

8. Dutra- Tomé L, Alencastro LS, Koller SH. A narrativa como proposta metodológica para o estudo do burnout. Psicologia \& Sociedade. 2014; 26(n. spe.): 107 - 116, doi: 10.1590/S010271822014000500012.

9. Pinheiro LRS, Farikoski C. Avaliação do Nível de Estresse de Policiais Militares. Revista de Psicologia da IMED. 2016; 8 (1): 14 - 19, doi: 10.18256/2175-5027/psico-imed.v8n1p14-19.

10. Ascari RA, Dumke M, Dacol PM, Junior SM, Sá CA, Lautert L. Prevalência de risco para síndrome de burnout em policiais militares. Cogitare enferm. 2016; 21 (2): 1 - 10, doi: 10.5380/ce.v21i2.44610. 
11. Albuquerque FJB, Melo CF, Araújo Neto JL. Avaliação da Síndrome de Burnout em Profissionais da Estratégia Saúde da Família da Capital Paraibana. Psicologia: Reflexão e Crítica. 2012; 25(3): 542 549, doi: 10.1590/S0102-79722012000300014.

12. Guimarães LAM, Mayer VM, Bueno HPV, Minari MRT, Martins LF. Síndrome de burnout e qualidade de vida de policiais militares e civis. Revista Sul Americana de Psicologia. 2014; 2(1): 98-122.

13. Rumin CR, Ferrari RAN, Barros GIF, Cardozo WR, Cavalhero RO. O Sofrimento Psíquico no Trabalho de Vigilância em Prisões. Psicol. cienc. prof. 2011; 31(1): 188-199, doi: 10.1590/S141498932011000100016.

14. Castro TCM, Merlo ARC. Reconhecimento e saúde mental na atividade de segurança pública. Psico. 2011; 42(4): 474-480.

15. Merlo ARC, Traesel ES, Baierle TC. Trabalho imaterial e contemporaneidade: um estudo na perspectiva da Psicodinâmica do Trabalho. Arquivos Brasileiros de Psicologia. 2011; 63(no. spe.): 94-104.

16. Guarda Municipal de Fortaleza. [Internet]. Prefeitura de Fortaleza. 2013 [Acesso em 09/07/2014]. Disponível em: http://www.fortaleza.ce.gov.br/gmf.

17. Maslach C, Jackson SE. Maslach Burnout Inventory. 2 ed. Palo Alto, CA: Consulting Psychologist Press; 1986.

18. Lautert L. O desgaste profissional: estudo empírico com enfermeiras que trabalham em hospitais. Rev Gaúcha Enferm. 1997; 18 (2): 133 - 144.

19. Cabral A, Zeni E. O trabalho com qualidade de vida: um estudo de caso na guarda municipal de chapecó. Revista Cientifica Tecnológica. 2016; 4(1): 175-194.

20. Da Silva-Junior JS, Fischer FM. Afastamento do trabalho por transtornos mentais e estressores psicossociais ocupacionais. Rev Bras Epidemiol. 2015; 18 (4): 735 - 744, doi: 10.1590/19805497201500040005.

21. Afonso JMP, Gomes AR. Stress ocupacional em profissionais de segurança pública: um estudo com militares da Guarda Nacional Republicana. Psicol. Reflex. Crit. 2009; 22 (2): 294 - 303, doi: 10.1590/S0102-79722009000200017.

22. Gonçalo H, Gomes AR, Barbosa F, Afonso J. Stresse ocupacional em forças de segurança: Um estudo comparativo. Análise Psicológica. 2010; 28 (1): 165 - 178.

23. Silveira NM, Vasconcellos SJL, Cruz LP, Kiles RF, Silva TP, Castilhos DG, Gauer GJC. Avaliação de burnout em uma amostra de policiais civis. Rev. psiquiatr. Rio Gd. Sul [Internet]. 2005; 27 (2): 159163, doi: 10.1590/S0101-81082005000200006. 